\title{
Corrosion Sensor Development for Condition-Based Maintenance of Aircraft
}

\author{
Gino Rinaldi, ${ }^{1}$ Trisha Huber, ${ }^{2}$ Heather McIntosh, ${ }^{3}$ Les Lebrun, ${ }^{3}$ \\ Heping Ding, ${ }^{3}$ and John Weber ${ }^{3}$ \\ ${ }^{1}$ NRC Institute for Aerospace Research, National Research Council Canada, Building M-14, Ottawa, ON, Canada K1A OR6 \\ ${ }^{2}$ Dockyard Laboratory Pacific, Defence Research \& Development Canada-Atlantic, CFB Esquimalt D199, \\ P.O. Box 17000, Station Forces, Victoria, BC, Canada V9A 7N2 \\ ${ }^{3}$ NRC Institute for Microstructural Sciences, National Research Council Canada, Building M-50, Ottawa, ON, Canada K1A OR6 \\ Correspondence should be addressed to Gino Rinaldi, gino.rinaldi@nrc-cnrc.gc.ca
}

Received 19 February 2012; Accepted 10 April 2012

Academic Editor: Peter Foote

Copyright () 2012 Gino Rinaldi et al. This is an open access article distributed under the Creative Commons Attribution License, which permits unrestricted use, distribution, and reproduction in any medium, provided the original work is properly cited.

\begin{abstract}
Aircraft routinely operate in atmospheric environments that, over time, will impact their structural integrity. Material protection and selection schemes notwithstanding, recurrent exposure to chlorides, pollution, temperature gradients, and moisture provide the necessary electrochemical conditions for the development and profusion of corrosion in aircraft structures. For aircraft operators, this becomes an important safety matter as corrosion found in a given aircraft must be assumed to be present in all of that type of aircraft. This safety protocol and its associated unscheduled maintenance requirement drive up the operational costs of the fleet and limit the availability of the aircraft. Hence, there is an opportunity at present for developing novel sensing technologies and schemes to aid in shifting time-based maintenance schedules towards condition-based maintenance procedures. In this work, part of the ongoing development of a multiparameter integrated corrosion sensor is presented. It consists of carbon nanotube/polyaniline polymer sensors and commercial-off-the-shelf sensors. It is being developed primarily for monitoring environmental and material factors for the purpose of providing a means to more accurately assess the structural integrity of aerospace aluminium alloys through fusion of multiparameter sensor data. Preliminary experimental test results are presented for chloride ion concentration, hydrogen gas evolution, humidity variations, and material degradation.
\end{abstract}

\section{Introduction}

Metals are vulnerable to corrosion due to environmental factors, and in the majority of cases engineering-grade metals and alloys are selected based on design and functionality priorities: strength, light-weight, mechanical and electrical properties, and so forth, rather than corrosion resistance per se [1]. Unfortunately, the cost of corrosion has a substantial impact on the economies of all nations with the United States spending approximately $\$ 437$ billion annually for corroded infrastructure maintenance and replacement [2].

This costing trend is increasing because aircraft are exposed to service operating environments that negatively impact their long-term durability. This is an on-going design, materials selection, and maintenance scheduling issue whose combined neglect or oversight can lead to a shortened life cycle or catastrophic failure of the aircraft $[3,4]$. For aircraft, the effects of corrosion damage are cumulative and can increase in severity if the damage is not detected early. A serious result of undetected corrosion is that it can have a cascading effect by which it can precipitate and accelerate fatigue damage [5] leading to a corresponding decrease in the load bearing capacity for that structure. In this respect, the structural integrity of the aircraft is undermined by two separate yet complicit factors. Corrosion damage occurs slowly, insidiously, and often out of sight. In the short term, it may represent only a small part of the total maintenance cost of a short-life aircraft; however, due to its cumulative nature, corrosion damage-based maintenance expenses will inflate to a substantial amount the ownership cost of a long-life aircraft at retirement [6].

Due to the extreme environments often encountered in military and search-and-rescue (SAR) air operations, these types of aircraft are often flown in conditions that can 


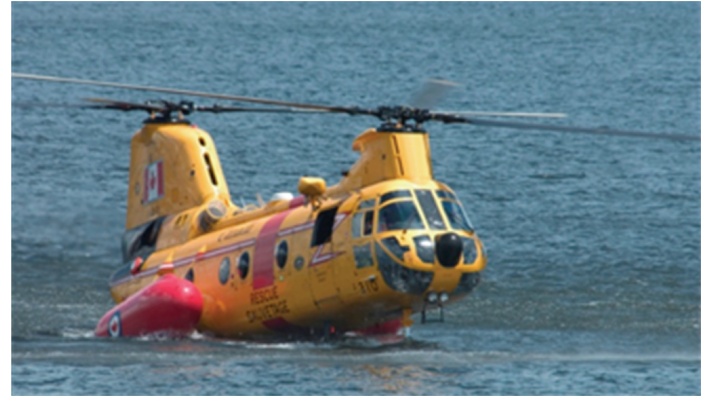

(a)

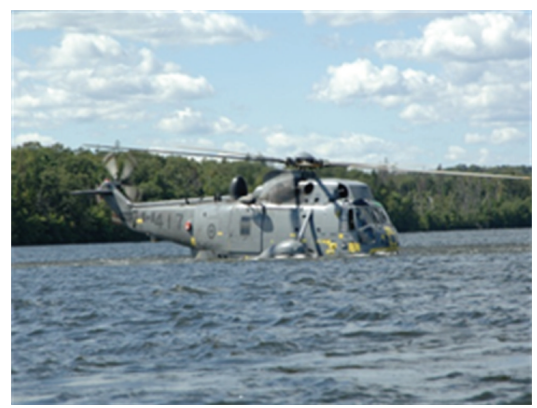

(c)

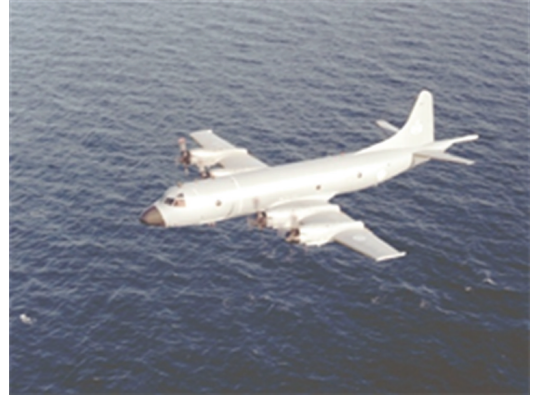

(b)

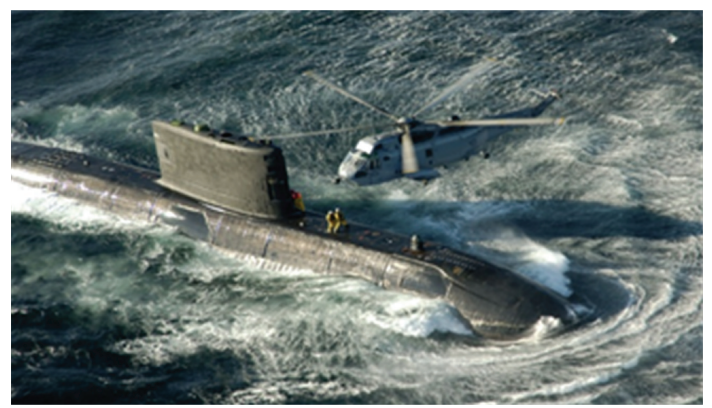

(d)

Figure 1: (a) an CH-113 "Labrador" search-and-rescue helicopter during a training exercise; (b) an CP-140 "Aurora" aircraft on patrol; (c) an CH-124 "Sea King" helicopter during water-bird training; (d) an CH-124 "Sea King" helicopter rendezvousing with HMCS Windsor.

be considered to be "pushing their design and operational limits." As an example, exposure of a few hours to sea water may cause pitting, and if it is not removed, as can happen in hidden or difficult to access areas, it can lead to premature crack nucleation in aircraft components [7]. Also, dissolved chlorides are known to be involved in the penetration of the protective aluminium oxide layer [8]. The combined effects of corrosion and accelerated fatigue due to corrosion will eventually limit the performance and availability of these aircraft. Presented in Figure 1 are images of Canadian Forces and SAR aircraft [9] in various typical operational modes.

The integrated corrosion sensor development program presented here intends to mitigate these consequences by providing a condition-based approach to aircraft maintenance protocols through a data-intensive maintenance scheduling methodology. The primary aim is to trim maintenance schedules down to actual structural, component or material requirements as opposed to operating under predefined inspection timetables. Presented in Figure 2 is a schematic of various factors involved in the corrosion process. Using data fusion, these factors will determine a corrosion index, which in turn will determine the conditionbased maintenance (CBM) protocol. Figure 3 shows a proposed breakdown of the CBM into preventative and corrective protocols as a function of the corrosion index.

\section{Aluminium Corrosion Chemistry in Aqueous Solution}

2.1. Aluminium Oxide Passivation Layer. Aluminium is only workable from an engineering point of view because of the existence of a natural aluminium oxide $\left(\mathrm{Al}_{2} \mathrm{O}_{3}\right)$ protective layer, without which it would react violently. Aluminium reacts quickly with any available oxygen source to immediately form $\mathrm{Al}_{2} \mathrm{O}_{3}$. This thin layer inhibits chemical interaction between bare aluminium and the local environment. However, aqueous solutions carrying anionic species can diffuse through $\mathrm{Al}_{2} \mathrm{O}_{3}$ and lead to corrosion [10]. The corrosion process of aluminium (Al) in aqueous solutions involves a combination of several factors: (i) moisture; (ii) anionic concentration (chloride); (iii) $\mathrm{pH}$ of the solution; (iv) temperature $[11,12]$.

The protective reactions, in air and water, respectively, are given as

$$
\begin{gathered}
4 \mathrm{Al}+3 \mathrm{O}_{2} \longrightarrow 2 \mathrm{Al}_{2} \mathrm{O}_{3}, \\
2 \mathrm{Al}+3 \mathrm{H}_{2} \mathrm{O} \longrightarrow 2 \mathrm{Al}_{2} \mathrm{O}_{3}+\left(6 \mathrm{H}^{+}+6 \mathrm{e}^{-} \longrightarrow 3 \mathrm{H}_{2}\right) .
\end{gathered}
$$

Equation (2) indicates that the reaction of $\mathrm{Al}$ with water $\left(\mathrm{H}_{2} \mathrm{O}\right)$ will also release hydrogen gas $\left(\mathrm{H}_{2}\right)$.

2.2. Oxidation Reduction. The oxidation reaction of $\mathrm{Al}$ (in $\mathrm{H}_{2} \mathrm{O}$ ) is given by [13]:

$$
\mathrm{Al} \longrightarrow \mathrm{Al}^{3+}+3 \mathrm{e}^{-}
$$

Trivalent $\mathrm{Al}$ ions $\left(\mathrm{Al}^{3+}\right)$ readily bind with chloride $\left(\mathrm{Cl}^{-}\right)$ and hydroxide $\left(\mathrm{OH}^{-}\right)$ions present in the solution, and this bonding competition either leads to continued degradation of the $\mathrm{Al}_{2} \mathrm{O}_{3}$ layer or its regeneration, respectively. 


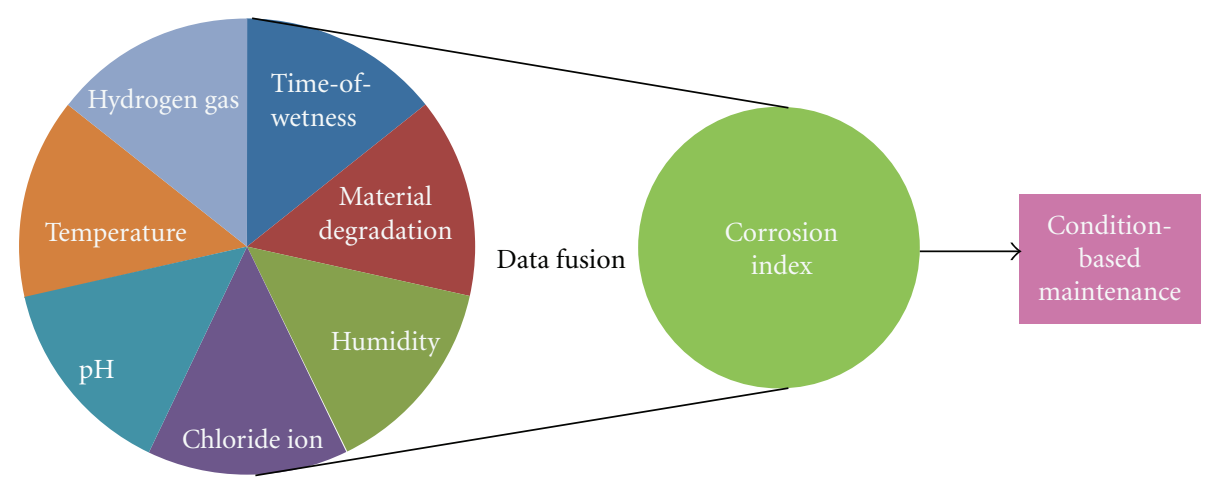

FIgURE 2: An overview of the proposed sensor nodes.

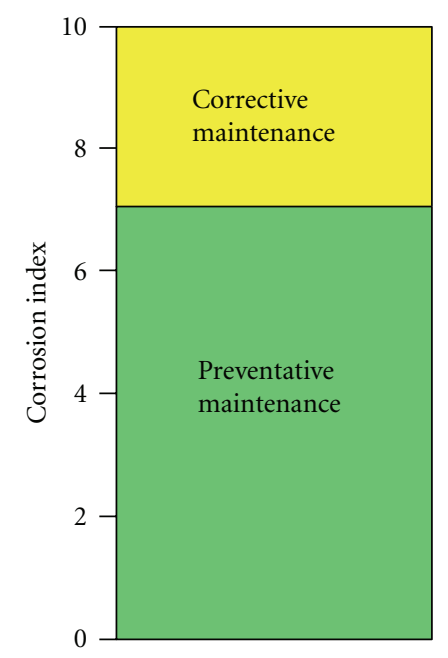

Figure 3: A sample CBM scheme based on an environmental factor derived corrosion index.

The above reaction is electrochemically balanced by a corresponding reduction reaction given by [13]:

$$
6 \mathrm{H}^{+}+6 \mathrm{e}^{-} \longrightarrow 3 \mathrm{H}_{2}
$$

where the hydronium $\left(\mathrm{H}^{+}\right)$ions are a result of the dissolution of $\mathrm{H}_{2} \mathrm{O}$ given by

$$
\mathrm{H}_{2} \mathrm{O} \longleftrightarrow \mathrm{H}^{+}+\mathrm{OH}^{-} .
$$

Generally, the dissolution of $\mathrm{Al}$ in water is the sum of the oxidation and reduction reactions, as given by (3) and (4):

$$
2 \mathrm{Al}+6 \mathrm{H}^{+}+6 \mathrm{e}^{-} \longrightarrow 2 \mathrm{Al}^{3+}+6 \mathrm{e}^{-}+3 \mathrm{H}_{2}
$$

Employing (5) and (6), the dissolution of $\mathrm{Al}$ with water results in the formation of aluminium-hydroxide $\left(\mathrm{Al}(\mathrm{OH})_{3}\right)$ and the release of $\mathrm{H}_{2}$ and is given by $[8,14,15]$ :

$$
2 \mathrm{Al}+6 \mathrm{H}_{2} \mathrm{O} \longrightarrow 2 \mathrm{Al}(\mathrm{OH})_{3}+3 \mathrm{H}_{2} .
$$

2.3. Chloride-Based Aqueous Solution. Chloride enters the aqueous solution through the absorption of gaseous hydrochloric acid or an organic gas containing chlorine or through the deposition of sea salt [16]. For chloride-based solutions, the oxidation/reduction reactions would involve the following [17]:

$$
\begin{gathered}
2 \mathrm{Cl}^{-} \longrightarrow \mathrm{Cl}_{2}+2 \mathrm{e}^{-}, \\
2 \mathrm{H}_{2} \mathrm{O} \longrightarrow \mathrm{O}_{2}+4 \mathrm{H}^{+}+4 \mathrm{e}^{-}\left(\text {or } 2 \mathrm{H}^{+}+2 \mathrm{OH}^{-}\right) .
\end{gathered}
$$

As more and more $\mathrm{Cl}^{-}$is adsorbed, repair of the $\mathrm{Al}_{2} \mathrm{O}_{3}$ layer is no longer possible, and corrosion proceeds rapidly $[12,18]$. This process is shown schematically in Figure 4(a). Competitive adsorption of $\mathrm{Cl}^{-}$, or $\mathrm{OH}^{-}$at $\mathrm{Al}^{3+}$ lattice sites will result in either continued dissolution or reoxidization, respectively [18-20]. A sample chain structure for a dissolution complex is shown in Figure 4(b) (adapted from [21]).

2.4. Hydrochloric Acid. Hydrochloric acid represents an aggressive environment for $\mathrm{Al}$, particularly $\mathrm{Al}$ alloys $[13,16$, 22]. After $\mathrm{Al}_{2} \mathrm{O}_{3}$ penetration by $\mathrm{Cl}^{-}$, the dissolution process continues as follows:

$$
\mathrm{AlCl}_{3}+3 \mathrm{H}_{2} \mathrm{O} \longrightarrow \mathrm{Al}(\mathrm{OH})_{3}+3 \mathrm{HCl},
$$

where $\mathrm{HCl}$ further attacks the $\mathrm{Al}_{2} \mathrm{O}_{3}$ layer:

$$
\mathrm{Al}_{2} \mathrm{O}_{3}+6 \mathrm{HCl} \longrightarrow 2 \mathrm{AlCl}_{3}+3 \mathrm{H}_{2} \mathrm{O}
$$

which exposes more bare aluminium to $\mathrm{HCl}$ and the evolution of $\mathrm{H}_{2}$ [23]:

$$
2 \mathrm{Al}+6 \mathrm{HCl} \longrightarrow 2 \mathrm{AlCl}_{3}+3 \mathrm{H}_{2} .
$$

When fully corroded, 1 gram of Al will produce 1.245 litres of $\mathrm{H}_{2}$ gas [23]. The $\mathrm{AlCl}_{3}$ in (9) can then further react with $\mathrm{H}_{2} \mathrm{O}$ to create more $\mathrm{HCl}$ (as in (11)), and the dissolution process continues. Of importance, during the corrosion process, $\mathrm{H}_{2}$ gas creates blisters on the $\mathrm{Al}$ surface due to $\mathrm{H}_{2}$ gas accumulating in microcavities beneath the $\mathrm{Al}_{2} \mathrm{O}_{3}$ layer, thereby causing structural damage [24]. 


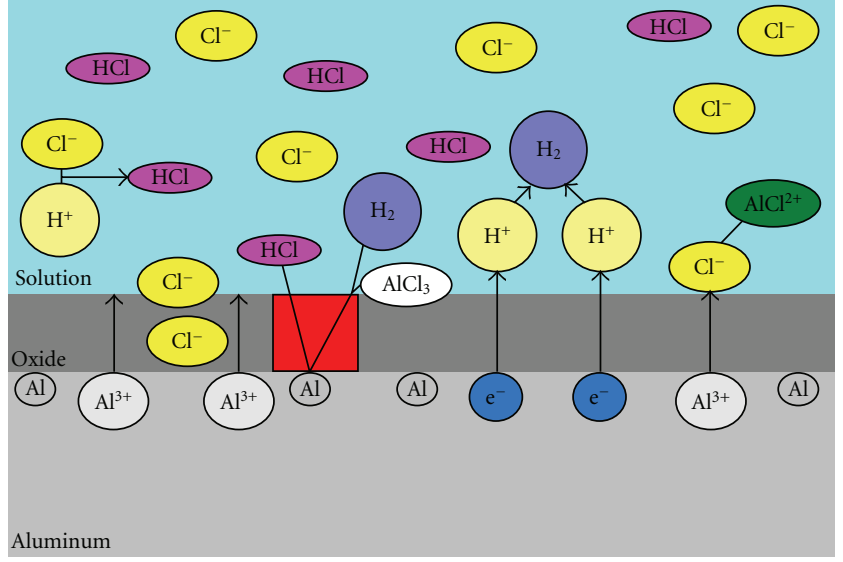

(a)

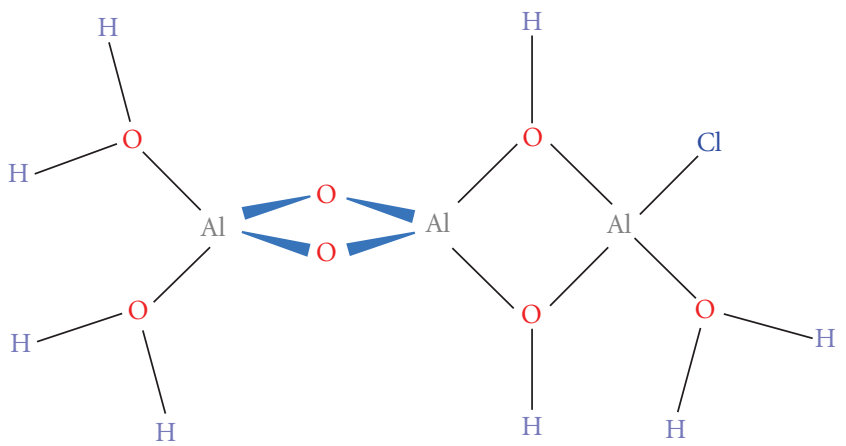

(b)

FIGURE 4: (a) breakdown of the aluminium oxide layer in a chloride-based aqueous medium. (b) chain structure showing the bonding of chloride with aluminium at a competition site.

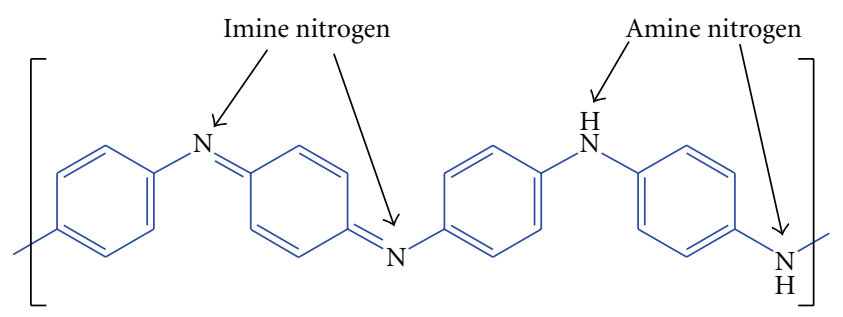

(a)

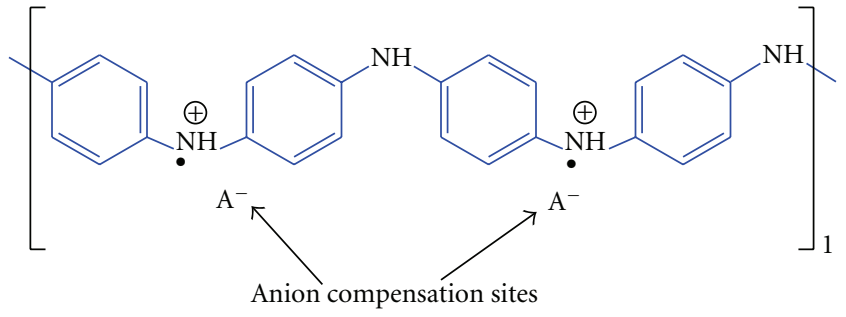

(b)

FIgURE 5: Chemical structure for the acid doping of PANI. (a) amine and imine nitrogen sites and (b) acid anion compensation sites.

\section{Sensor Node Development}

3.1. Hydrogen Gas and Humidity Sensing Nodes: Conducting Polymer-Based Sensing. Conducting polymers become electrically conductive through the addition or removal of $\pi$-electrons; however, polyaniline [25] (PANI) is more commonly doped by the addition of an acid. Shown in Figures 5(a) and 5(b) is a sample acid-doped chemical structure of PANI $[26,27]$. The electrical conductivity of PANI depends on the size and shape of the acid anion [27], and it may be further enhanced by incorporating carbon nanotubes (CNTs) in the preparation of the polymer [28]. The addition of carbon nanotubes is thought to influence the semiconductor charge carrier mechanism ( $p$-doped or $n$ doped) in polyaniline. Carbon nanotubes also significantly increase the current output of $I-V$ type tests [29] because CNTs exhibit greater electrical conductivity along their length as compared to conducting polymers, although bulk CNTs do not conduct as well as expected, as the charge transfer is believed to occur by hopping. The conductivity of composites apparently exceeds that of the parent materials alone. This apparent synergy is attributable to the reduction in contact resistance between CNTs by the presence of the conducting polymer. The PANI/CNTs blend can then be readily applied to a sensing platform such as interdigitated

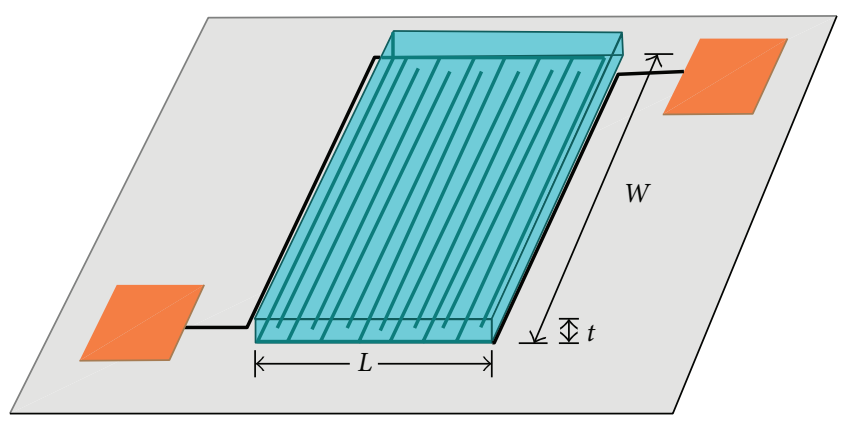

FIGURE 6: Schematic of an IDEs chip with a PANI polymer conductive coating.

electrodes (IDEs). Shown in Figure 6 is a schematic of an IDEs chip coated with PANI/CNTs (not to scale).

3.2. Integrated Chloride Ion, Relative Humidity, and Temperature Sensing Node. The development of this node presents an opportunity for integrating various "corrosion" sensors within the same enclosure. Also, the electronics and the associated computer interface capability being developed in parallel will provide a means that can allow for simultaneous 


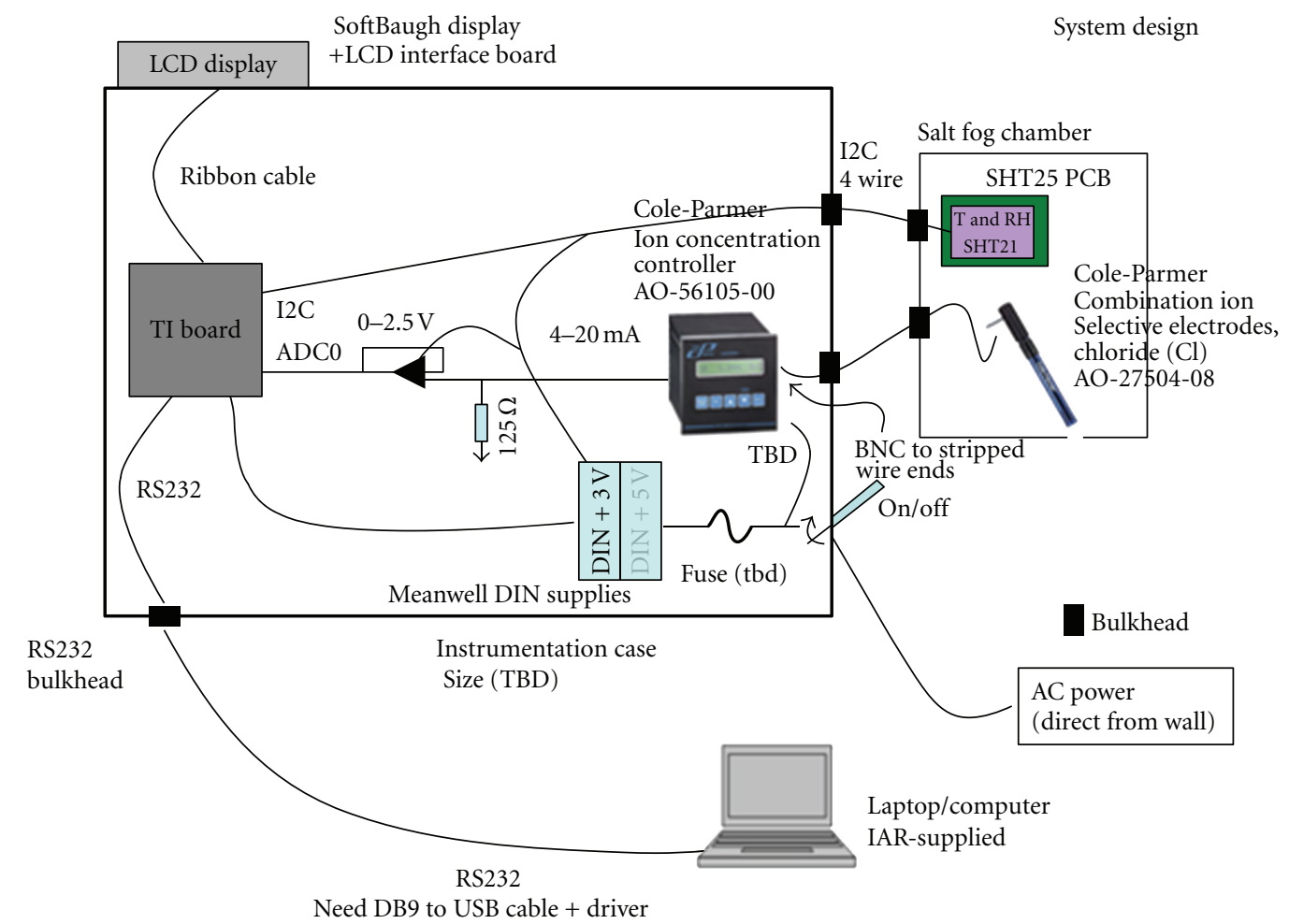

FIGURE 7: An overview of the components of the chloride ion, relative humidity, and temperature sensing node.

data acquisition from the various sensors. At this stage of the corrosion sensor project, it can be considered as a prototype from which refinements in sensor integration, electronics and scaling-down can be developed. This unit consists of COTS chloride ion, relative humidity, and temperature sensors. Currently, the primary components include (i) a chloride ion electrode (Cole-Parmer Instrument Company); (ii) an ion concentration controller that interfaces to the electrode (B\&C Electronics IC 7685); (iii) a packaged SHT21 humidity and temperature sensor (Sensirion AG); (iv) an MSP430FG461x/F20xx Experimenter's Board (Texas Instruments). Presented in Figure 7 is an overview of the integrated sensor. The microcontroller communicates with the SHT21 sensor through an I2C bus and reads the chloride ion level detected by the IC 7685 controller through one of its analog inputs (ADC0). The microcontroller platform is MSP430FG4618 (Texas Instruments). The firmware resides in the FLASH memory of the microcontroller device and performs various functions required for detection of chloride ion, temperature, and relative humidity. On power up, the system boots automatically and enters the default mode for detection.

At the current developmental stage of the corrosion sensor project, the microcontroller hardware and firmware are very much underutilized. For example, only one of the eleven available analog inputs is used, and the firmware is often in "sleep" mode. Thus, such a microcontroller system has room to accommodate future extensions such as adding more sensing elements, and more functions. In this respect, the integration of additional features along with size and weight reductions forms the basis for the next phase of the project.

3.3. Material Degradation Sensing Node. For the material degradation sensing node, an XCorr multiarray sensor (Aginova Inc.) was adopted. This sensor is a 16-element passive electrochemical sensor that does not require any applied voltage for operation (Figure 8(a)). The sensor's 16 elements are designed to measure uniform or localized corrosion through a naturally occurring corrosion-current between anodic and cathodic sites on the metal surface when it is exposed to a corrosive solution. The probe's 16 elements are connected together through a common interface within the electronics (Figure 8(b)). The corrosive aqueous solution creates both cathodic and anodic sites at the elements on the probe head (Figure $8(\mathrm{c})$ ). In this regard, corrosion at anodic sites can develop as on an actual metal surface. The system integrates data from the 16 individual elements and extrapolates both average and maximum yearly corrosion rates (Faraday's Law).

\section{Experimental Section}

4.1. Polyaniline Preparations. In its finished form, PANI/CNTs blends resemble a dark powder, as shown in Figure 9(a). In this state, it cannot be employed directly for sensing applications but must be dispersed in a suitable solvent in order to apply it to a sensor surface such as IDEs. In this work, two types of metallic IDEs (Synkera Technologies 


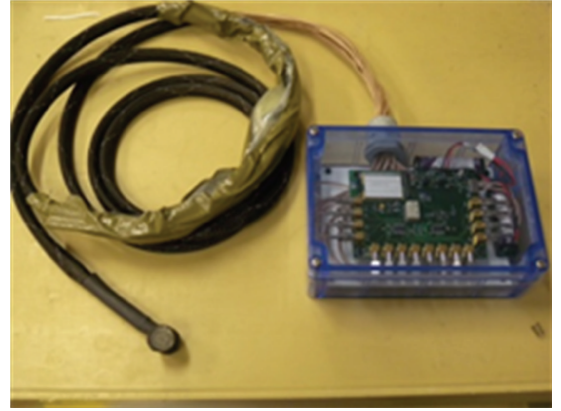

(a)

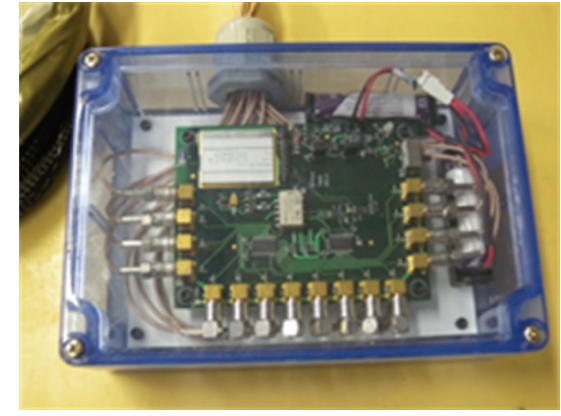

(b)

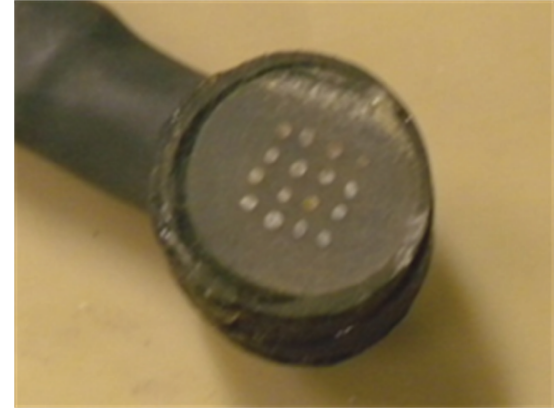

(c)

FIGURE 8: (a) XCorr multiarray sensor; (b) encased sensor electronics unit; (c) close-up of the 16 element probe.

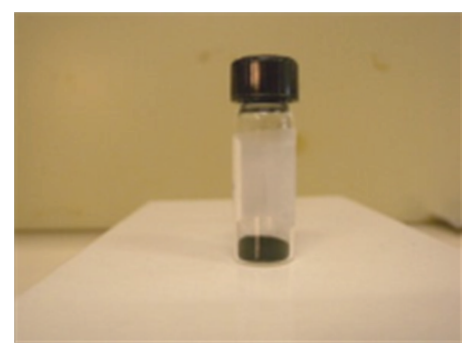

(a)

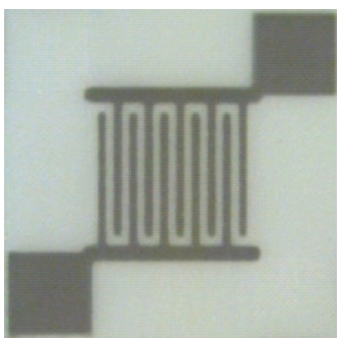

(b)

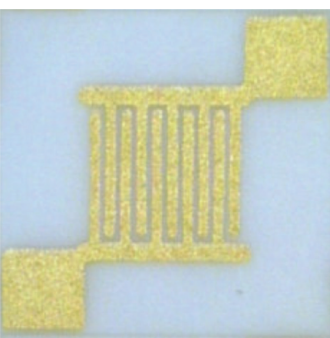

(c)

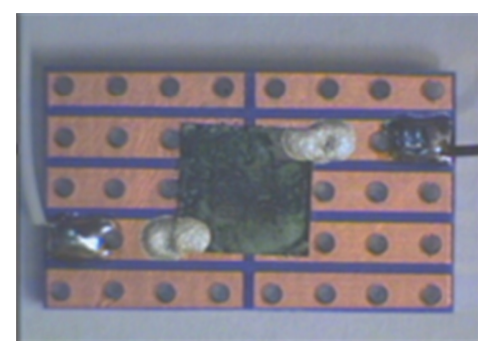

(d)

FIGURE 9: (a) PANI/CNTs “powder;” (b) platinum IDEs; (c) gold IDEs; (d) IDEs coated with a PANI/CNT blend.

Inc.), platinum (Figure 9(b)) and gold (Figure 9(c)), were employed for hydrogen gas and humidity sensing, respectively. Figure $9(\mathrm{~d})$ shows a platinum $5 \times 5 \mathrm{~mm}^{2}$ IDEs chip coated with a PANI/CNTs blend. In this figure, the IDEs chip is mounted onto a small electrical bread-board for ease of wiring and handling.

4.1.1. PANI: Hydrogen Gas Sensor Node. Hydrogen sensing using PANI and PANI/CNTs blends has been reported in the literature [30-32]. In this work, camphor sulfonic acid (CSA) was employed as the doping material. The PANI/CSA/CNTs were dispersed in a chloroform solvent $(0.040$ grams PANI/CNTs powder per $\mathrm{mL}$ solvent). The mixture was then placed into a sonication bath for approximately 18 hours in order to obtain a uniform suspension. Small pipettes were used to transfer the solutions, and drop small amounts onto platinum IDEs. Spin-coating (2000 RPM for 1 minute) was used to distribute the solution over the IDEs surface.

4.1.2. PANI: Humidity Sensor Node. Humidity sensing using PANI blends has been reported in the literature [3335]. In this work, the PANI humidity sensitization was prepared by doping PANI with bis-(2-ethylhexyl) phosphoric acid (HDEHP), toluene, and CNTs. For this preparation, ethanol was employed as a dispersing solvent (0.021 grams PANI/CNTs powder/ $0.5 \mathrm{~mL}$ solvent). The same transfer, sonication, and spin-coating procedures, as described previously, were employed. For humidity sensing, a gold IDEs chip was used.
4.1.3. Electrical Continuity Measurements. The electrical continuity of the deposited polymer was measured by applying a ramp voltage $(0-500 \mathrm{mV})$ across the polymer-coated IDEs and measuring the resulting current. A sample result is shown in Figure 10(a). The equation relating resistance $(R)$, length $(L)$, cross-sectional area $(A)$, and resistivity $(\rho)$ of an electrical conductor is given as

$$
R=\frac{\rho L}{A} .
$$

Due to the nature of the spin-coating polymer deposition process, $L$ and $A$ can vary between sensors. A sample conductivity curve is shown in Figure 10(b).

Given in Table 1 are the electrical continuity characteristics for the various IDEs/polymer sensors tested. The resistances shown include the contact resistance of the pads, associated wiring, and the particular PANI formulation. For ease of comparison, in this table the conductivity ${ }_{100}$ is a derived value for a polymer thickness of 100 microns.

\subsection{Experimental Results}

4.2.1. PANI/CSA Hydrogen Sensing. The PANI/CSA-based sensors developed for hydrogen sensing were tested in a dry hydrogen/argon atmosphere. Presented in Figure 11 is an overview of the fully automated gas flow system and electrical interface. Data was acquired in real time and transferred to a PC (not shown). Shown in Figure 12 is a typical response obtained for the hydrogen sensors. It can be seen from 


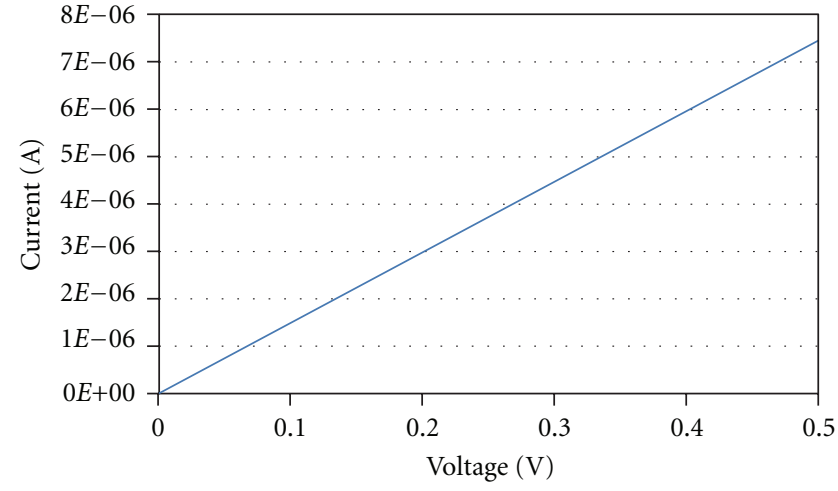

(a)

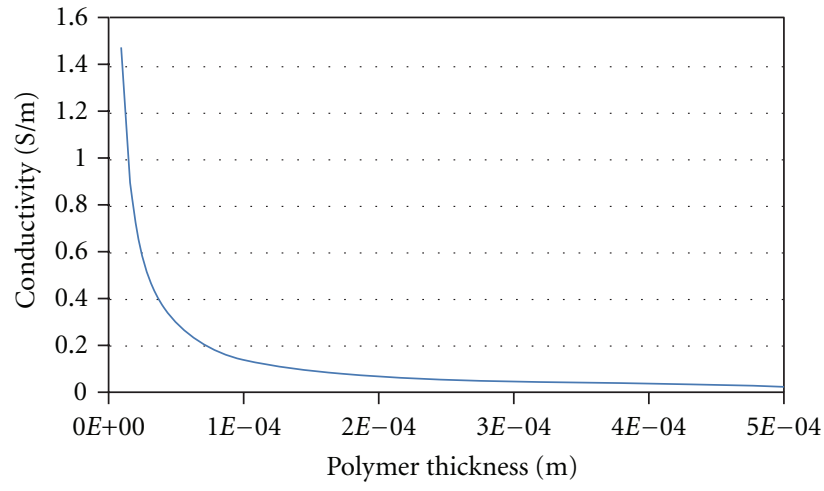

(b)

Figure 10: Continuity testing. (a) current as a function of an applied ramp voltage and (b) electrical conductivity as a function of the polymer thickness.

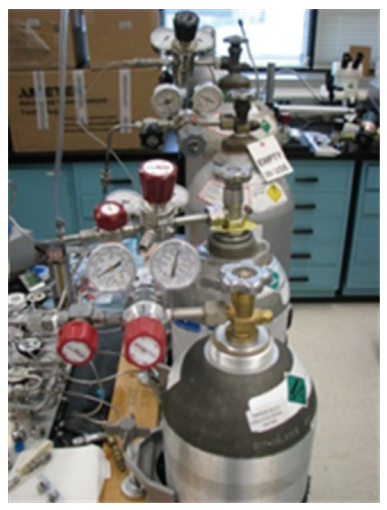

(a)

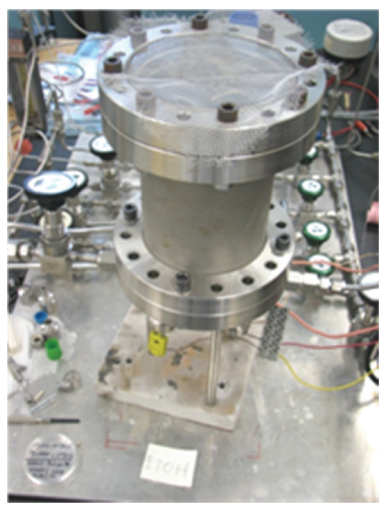

(b)

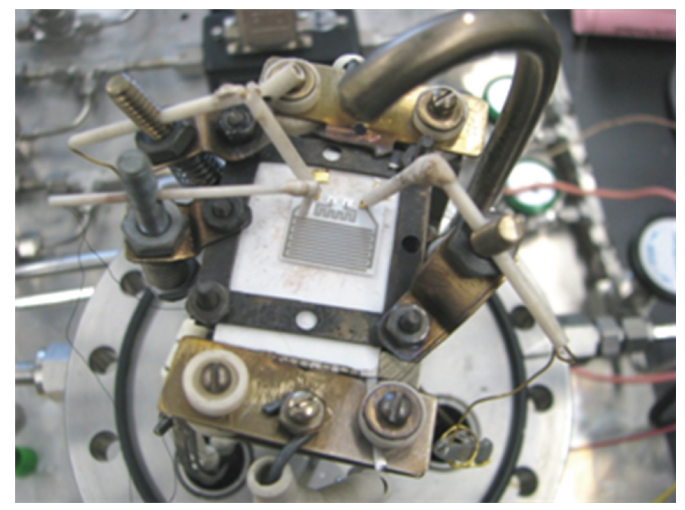

(c)

FIGURE 11: (a) argon and hydrogen gas cylinders; (b) the hydrogen/argon gas chamber used for the PANI sensor testing; (c) interior of the chamber and electrical contacts.

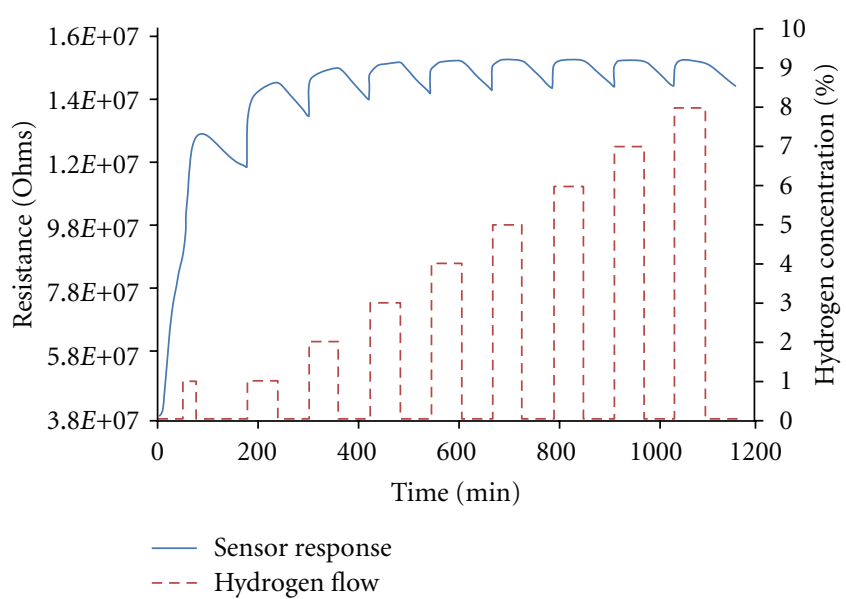

FIGURE 12: PANI hydrogen sensor response as a function of hydrogen gas concentration (dashed line).

this figure that the PANI/CSA formulation is sensitive to the presence of hydrogen. However, it is summarized that
TABLE 1: The measured resistances and derived conductivities for various polyaniline polymer formulations.

\begin{tabular}{lcc}
\hline Sensor designations & $\begin{array}{c}\text { Resistance } \\
\left(10^{3} \text { ohms }\right)\end{array}$ & $\begin{array}{c}\text { Conductivity } \\
(\mathrm{S} / \mathrm{m})\end{array}$ \\
\hline CSA-1 & 68 & 0.147 \\
CSA-2 & 758.1 & 0.0132 \\
CSA-3 & 4.1 & 2.42 \\
CSA/CNTs-1 & 4.0 & 2.46 \\
CSA/CNTs-2 & 2.0 & 4.93 \\
HDEHP-1-A & 377.6 & 0.0265 \\
HDEHP-1-B & 91.1 & 0.11 \\
HDEHP-2-A & 102.1 & 0.098 \\
HDEHP-2-B & 105.1 & 0.095 \\
HDEHP-3-A & 28.6 & 0.35 \\
HDEHP-3-B & 7.2 & 1.39 \\
HDEHP-4-A & 0.009 & 1000 \\
HDEHP-4-B & 0.011 & 882 \\
\hline
\end{tabular}

the similar responses seen for different hydrogen concentrations are in part due to hydrogen-bonding sites within 


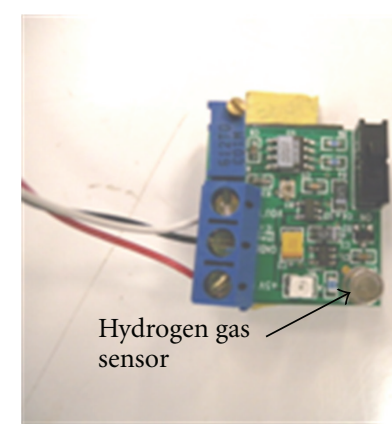

(a)

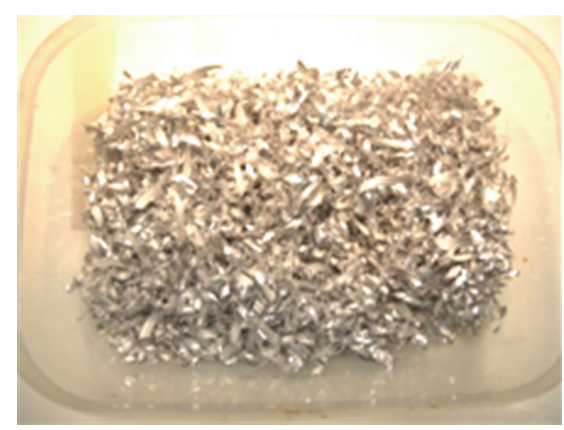

(b)

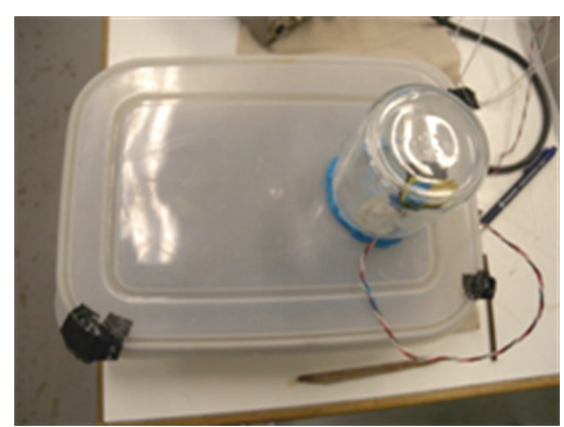

(c)

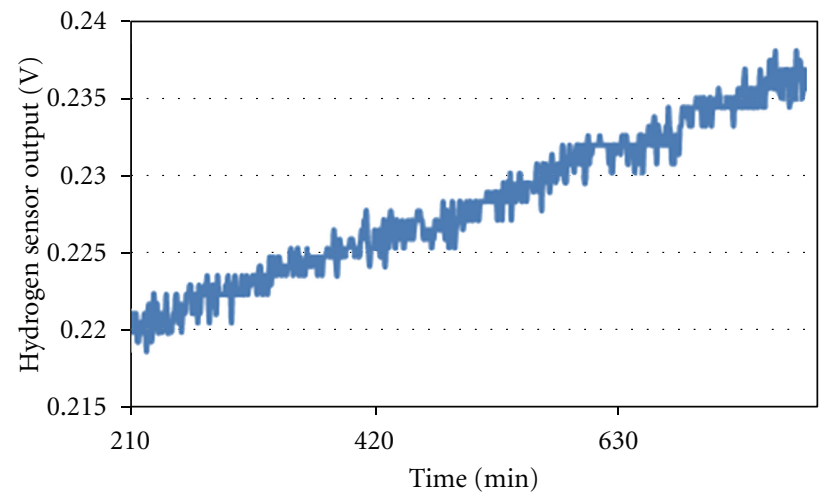

(d)

FIGURE 13: (a) hydrogen sensor and evaluation circuit board; (b) 6061-T6 aluminium alloy shards in a plastic container; (c) overview of the test setup showing the glass jar with hydrogen sensor within; (d) the sensor response as a function of hydrogen evolution over time.

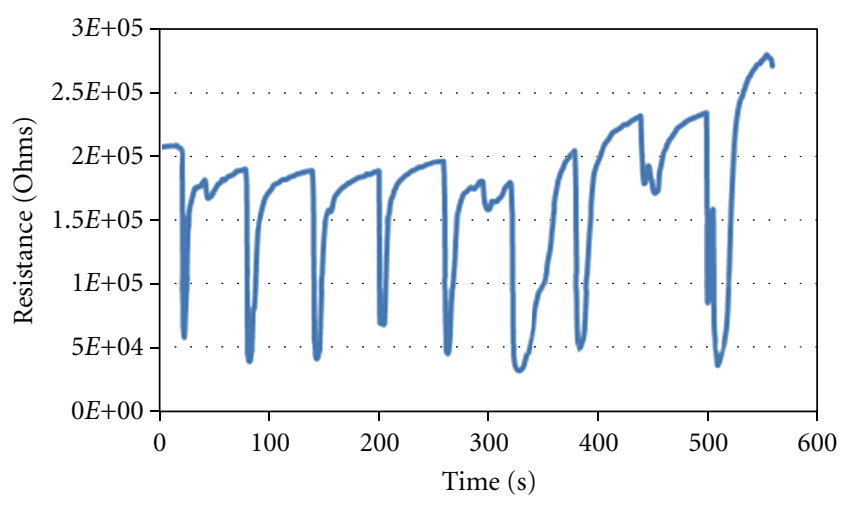

(a)

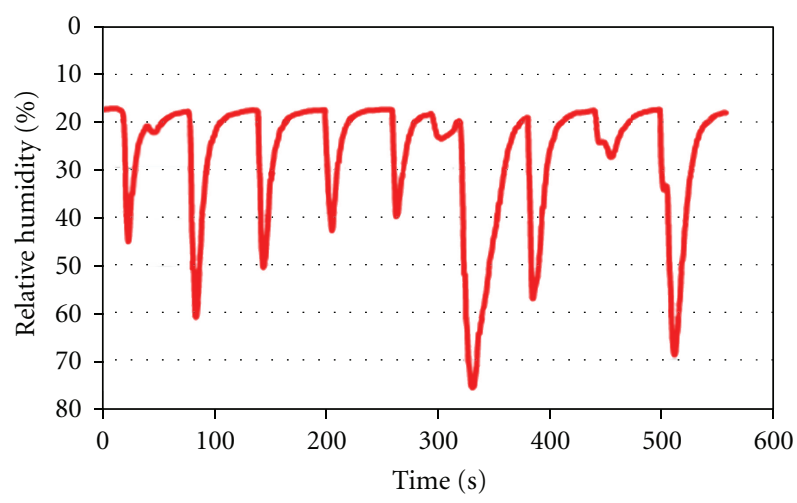

(b)

FIGURE 14: Humidity sensor responses. (a) PANI-based humidity sensor and (b) COTS humidity sensor.

the PANI/CSA material not being fully vacated when the flow is stopped or it is possible that the saturation point for this particular PANI/CSA formulation is below $1 \%$ hydrogen. Another possibility is that despite curing of the polymer in a dessicator prior to testing, some residual moisture may have remained trapped, thereby limiting the effective sensitivity of the PANI/CSA in these tests.

4.2.2. COTS Hydrogen Sensor. Described here is an experiment in which hydrogen gas was detected evolving from 6061-T6 aluminium alloy in a 5\% saltwater solution. This test was carried out to demonstrate the possibility of measuring hydrogen evolution for corrosion monitoring of aluminium. Testing was carried out with a COTS hydrogen sensor (Kebaili Corporation) as shown in Figure 13(a). This sensor is capable of measuring hydrogen up to $4 \%$ per volume in air and is based on the microcatalytic oxidation of hydrogen. The variation of the sensing element resistance due to the exothermic reaction of hydrogen oxidation is directly proportional to the hydrogen concentration. In these tests, the baseline offset of the sensor was set to $215 \mathrm{mV}$. These "accelerated" corrosion tests consisted of immersing 


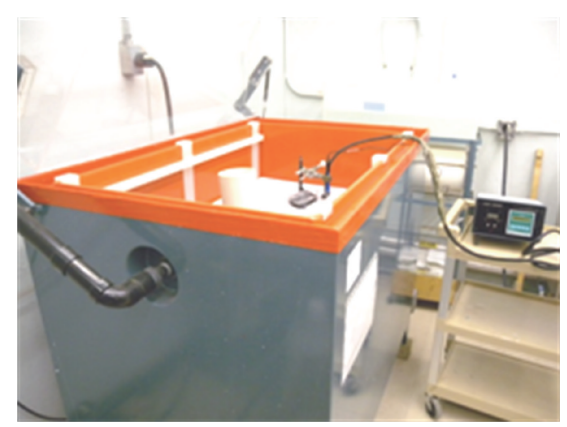

(a)

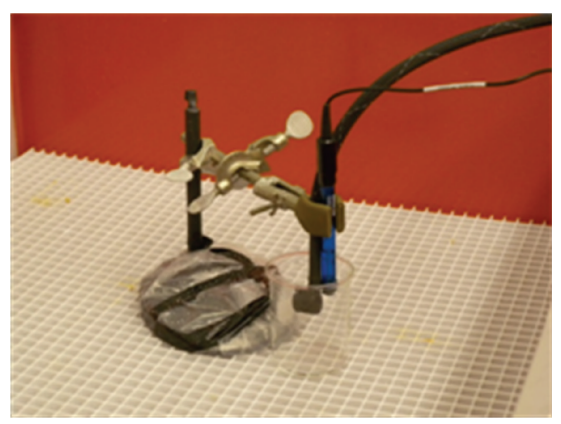

(b)

FIGURE 15: (a) salt-fog chamber and (b) chloride ion and material degradation probes.

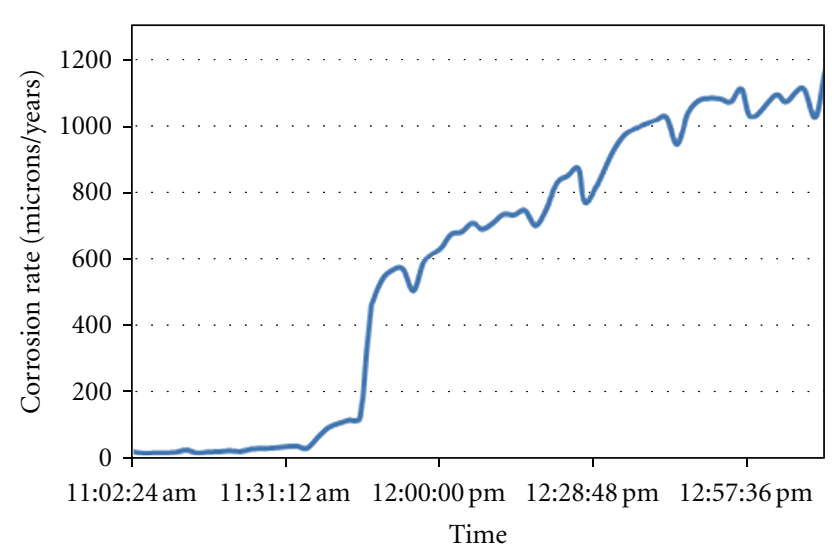

(a)

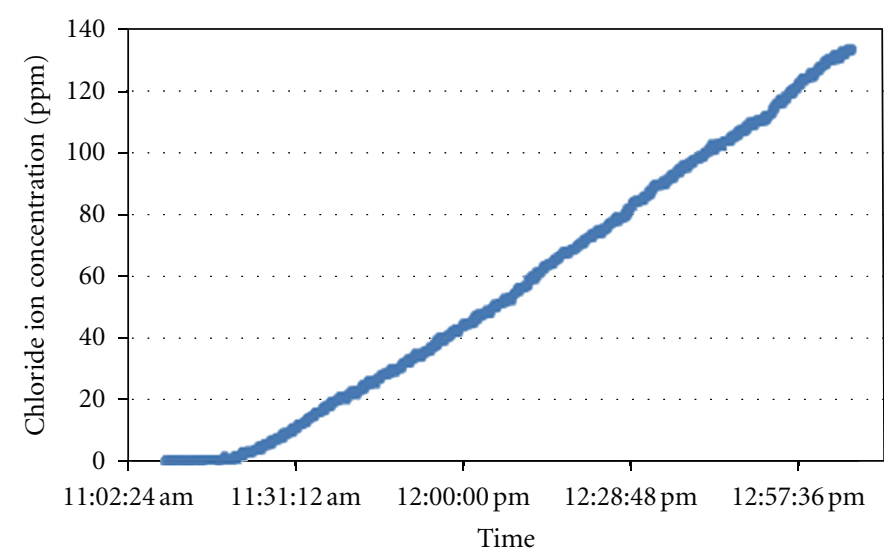

(b)

FIgURE 16: A comparison of the corrosion rate for steel with the increase in chloride ion concentration over time.

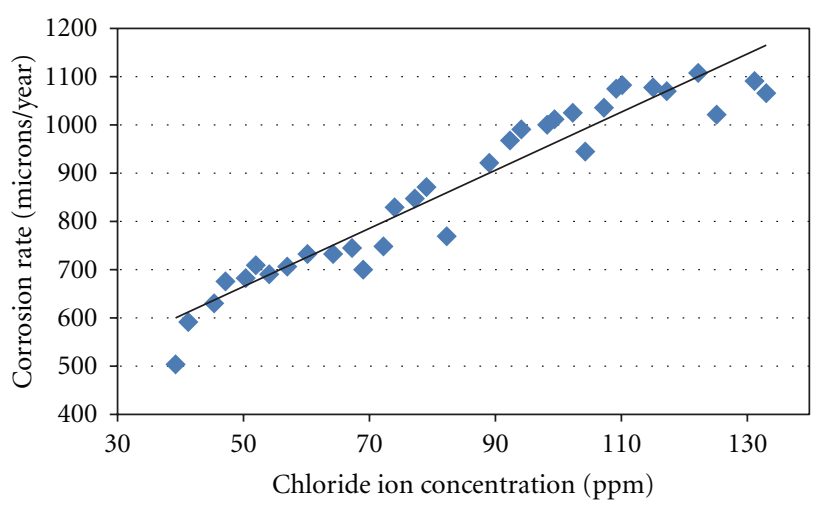

FIgURE 17: Corrosion rate as a function of the chloride ion concentration (solid line is a linear fit to the data).

6061-T6 aluminium alloy shards (Figure 13(b)) into a 5\% salt water solution. The hydrogen sensor was affixed inside a $250 \mathrm{~mL}$ glass jar and then placed over a small opening in the plastic cover (Figure 13(c)). The glass/plastic interface was sealed with a putty material. The aluminium was left in the saltwater solution for $\sim 36$ hours prior to testing. A digitized acquisition system (Omega instruNet) was employed for the data acquisition. The result, shown in Figure 13(d), shows a gradual increase in the hydrogen concentration over time. In this figure, the sensor output increased by $15 \mathrm{mV}$ over the measuring span, which corresponds to $\sim 3 \%$ hydrogen $(7.5 \mathrm{~mL})$ within the glass container, which in turn indicates that approximately $6 \mathrm{mg}$ of aluminium was consumed.

For aircraft applications, hydrogen sensing would require an enclosed area, where the diffusion of hydrogen is reduced and the gas concentrations reach levels that are practical for detection. Enclosed fuel storage areas such as under-fuel bladders, where condensation can form and initiate corrosion, are seen as potential areas for monitoring hydrogen gas evolution.

4.2.3. Humidity Sensor Node. Humidity sensor tests were conducted using a small container that allowed the flow of humid air through it. Both a PANI/HDEHP + toluene formulation IDEs sensor and a COTS (Omega RH820) sensor were placed inside the container for humidity sensing. Presented in Figure 14 is a comparison between the humidity sensitive response of the PANI-based and the COTS sensor as a function of time. As can be seen, the PANI sensor responds very well to humidity with excellent response.

4.2.4. Chloride Ion and Material Degradation Sensor Nodes. The chloride ion and material degradation testing was 
carried out in a salt-fog chamber (Singleton Corporation) as shown in Figure 15(a). The chloride ion sensor and material degradation sensor were placed into a beaker of deionized water (Figure 15(b)). Salt-fog condensate $(3.5 \%$ sodium chloride solution) was captured within the beaker and mixed with the deionized water. Data was acquired over a two-hour span. Shown in Figure 16 is a comparison of the increase in chloride concentration and material degradation as a function of time.

Shown in Figure 17 is the dependence of the yearly corrosion rate on chloride ion concentration $(40-130 \mathrm{ppm}$ range).

From Figures 16 and 17, it can clearly be seen that salt-fog condensation into the prepared beaker containing deionized is detected by the increase of $\mathrm{Cl}^{-}$concentration and also in the increase in the corrosion rate of test metal electrodes. For example, a $\mathrm{Cl}^{-}$concentration of $\sim 90 \mathrm{ppm}$ corresponds to a corrosion rate of $900 \mathrm{microns} /$ year. Hence, if such a solution were to remain undetected for an extended period of time, it could cause significant damage to an aircraft structure.

With the cumulative data of the various sensors, it will be possible to cross-correlate the influence of each corrosion parameter. In this way a theoretical corrosion index can be developed. In this regard, further testing is required in order to obtain adequate data for a more in-depth analysis and interpretation. This being said, the corrosion index will provide an indication of the environmental and material conditions so that either preventative or corrective maintenance actions can be taken.

At this stage the sensors are still in the developmental stage. However, as further refinement to the sensing technology is conducted, it is the aim that their use in aircraft will be in areas most prone to corrosion such as the cockpit in fighter aircraft, galley and latrine areas, or any other area prone to the accumulation and retention of moisture.

\section{Conclusions}

Part of the ongoing development of a multiparameter integrated corrosion sensor, consisting of carbon nanotube/polyaniline-based and commercial-off-the-shelf sensors, has been presented. This paper also described the integration methodology being investigated for the various sensor nodes and presented preliminary chloride ion, humidity, and temperature data acquisition capabilities. Also, experimental test results were presented for chloride ion concentration, hydrogen gas evolution, humidity variations, and material degradation. The integrated corrosion sensor development program presented here intends to provide a condition-based approach to aircraft maintenance protocols through a data-intensive maintenance scheduling methodology in order to tailor maintenance schedules for structural/component/material requirements as opposed to operating under predefined inspection timetables. Through data fusion, environmental and material factors were proposed for determining a corrosion index, which in turn was suggested for developing condition-based maintenance protocol consisting of both preventative and corrective maintenance scheduling.

\section{Acknowledgments}

The technical support given by Dr. Gerardo Diaz-Quijada and Dr. Bhavana Deore from NRC Steacie Institute for Molecular Sciences, and Dr. Jim Tunney and Dr. Jeffrey Dunford from NRC Institute for Chemical Process and Environmental Technology is gratefully acknowledged.

\section{References}

[1] C. M. Hansson, "The impact of corrosion on society," Metallurgical and Materials Transactions A, vol. 42, no. 10, pp. 2952-2962, 2011.

[2] A. Winkleman, E. B. Svedberg, R. E. Schafrik, and D. J. Duquette, "Preventing corrosion from wearing our future away," Advanced Materials and Processes, vol. 169, no. 3, pp. 26-31, 2011.

[3] N. Symonds and C. Pitt, "Military helicopters: have the seeds of future accidents already been sown?" Engineering Failure Analysis, vol. 13, no. 3, pp. 493-515, 2006.

[4] Accident Report: Aloha Airlines, "Flight 243, national transportation safety board,” Tech. Rep. NTSB/AAR-89/03, 1989.

[5] A. Jaya, U. H. Tiong, and G. Clark, "The interaction between corrosion management and structural integrity of aging aircraft," Fatigue and Fracture of Engineering Materials and Structures, vol. 35, no. 1, pp. 64-73, 2012.

[6] W. Wallace, D. W. Hoeppner, and P. V. Kandachar, "AGARD corrosion handbook volume 1-aircraft corrosion: causes and case histories," Tech. Rep. AGARD-AG-278, North Atlantic Treaty Organization, 1985.

[7] R. M. Chlistovsky, P. J. Heffernan, and D. L. DuQuesnay, "Corrosion-fatigue behaviour of 7075-T651 aluminum alloy subjected to periodic overloads," International Journal of Fatigue, vol. 29, no. 9-11, pp. 1941-1949, 2007.

[8] G. Rinaldi, "Chloride ion and humidity sensor for aluminum corrosion monitoring: sensor technology selection and overview," Defence R\&D Canada, Technical Note DRDCAtlantic TN 2009-209, 2009.

[9] http://www.rcaf-arc.forces.gc.ca/v2/netpub/index-eng.asp? mode $=1$.

[10] K. S. Lewis, J. Yuan, and R. G. Kelly, "Chemical conditions inside occluded regions on corroding aircraft aluminum alloys," Journal of Chromatography A, vol. 850, no. 1-2, pp. 375-380, 1999.

[11] A. Tosun, M. Ergun, M. Balbaşi, and M. Tamirci, "The use of experimental design method for the investigation of pitting potential of aluminum," Materials and Corrosion, vol. 58, no. 7, pp. 502-505, 2007.

[12] K. F. Lorking and J. E. O. Mayne, "The corrosion of aluminium," Journal of Applied Chemistry, vol. 11, pp. 170180, 1961.

[13] C. Vargel, Corrosion of Aluminium, Elsevier, 2004.

[14] L. Soler, J. Macanás, M. Muñoz, and J. Casado, "Aluminum and aluminum alloys as sources of hydrogen for fuel cell applications," Journal of Power Sources, vol. 169, no. 1, pp. 144149, 2007.

[15] J. Skrovan, A. Alfantazi, and T. Troczynski, "Enhancing aluminum corrosion in water," Journal of Applied Electrochemistry, vol. 39, no. 10, pp. 1695-1702, 2009.

[16] T. E. Graedel, "Corrosion mechanisms for aluminum exposed to the atmosphere," Journal of the Electrochemical Society, vol. 136, no. 4, pp. 204C-212C, 1989. 
[17] T. Urabe, M. Tanaka, S. Kumakura, and T. Tsugoshi, "Study on chemical speciation in aluminum chloride solution by ESI-QMS," Journal of Mass Spectrometry, vol. 42, no. 5, pp. 591-597, 2007.

[18] A. A. Mazhar, W. A. Badawy, and M. M. Abou-Romia, "Impedance studies of corrosion resistance of aluminium in chloride media," Surface and Coatings Technology, vol. 29, no. 4, pp. 335-345, 1986.

[19] R. M. Stevanović, A. R. Despić, and D. M. Dražić, "Activation of aluminium in chloride containing solutions," Electrochimica Acta, vol. 33, no. 3, pp. 397-404, 1988.

[20] T. H. Nguyen and R. T. Foley, "The chemical nature of aluminum corrosion: II. The initial dissolution step," Journal of the Electrochemical Society, vol. 129, no. 1, pp. 27-32, 1982.

[21] A. Sarpola, V. Hietapelto, J. Jalonen, J. Jokela, and R. S. Laitinen, "Identification of the hydrolysis products of $\mathrm{AlCl}_{3}$ $6 \mathrm{H}_{2} \mathrm{O}$ by electrospray ionization mass spectrometry," Journal of Mass Spectrometry, vol. 39, no. 4, pp. 423-430, 2004.

[22] H. W. von Kohlschütter and P. Hantelmann, "Basiche Aluminiumchloride," Zeitschrift für Anorganische und Allgemeine Chemie, vol. 248, pp. 319-344, 1941 (German).

[23] A. Reza, A. Kemal, and P. E. Markey, "Runaway reactions in aluminum, aluminum chloride, $\mathrm{HCl}$, and steam: an investigation of the 1998 CONDEA vista explosion in Maryland," Process Safety Progress, vol. 21, no. 3, pp. 261-267, 2002.

[24] S. N. Rashkeev, K. W. Sohlberg, S. Zhuo, and S. T. Pantelides, "Hydrogen-induced initiation of corrosion in aluminum," Journal of Physical Chemistry C, vol. 111, no. 19, pp. 71757178, 2007.

[25] A. G. Macdiarmid, J. C. Chiang, A. F. Richter, and A. J. Epstein, "Polyaniline: a new concept in conducting polymers," Synthetic Metals, vol. 18, no. 1-3, pp. 285-290, 1987.

[26] T. A. Huber, "A literature survey of polyaniline, Part 1: polyaniline as a radar absorbing material," Defence R\&D Canada, Technical Memorandum DRDC-Atlantic TM 2003014, 2003.

[27] T. Huber, P. Saville, and D. Edwards, "Investigations into the polyaniline and polypyrrole families of conducting polymers for application as radar absorbing materials," Defence R\&D Canada, Technical Memorandum DRDC-Atlantic TM 2003005, 2003.

[28] C. Chow and T. Huber, "The potential of conducting polymer-carbon nanotube composites as supercapacitor electrodes," Defence R\&D Canada, Technical Memorandum DRDC-Atlantic TM 2007-320, 2007.

[29] P. C. Ramamurthy, A. M. Malshe, W. R. Harrell, R. V. Gregory, K. McGuire, and A. M. Rao, "Polyaniline/singlewalled carbon nanotube composite electronic devices," SolidState Electronics, vol. 48, no. 10-11, pp. 2019-2024, 2004.

[30] C. Conn, S. Sestak, A. T. Baker, and J. Unsworth, "A Polyaniline-based selective hydrogen sensor," Electroanalysis, vol. 10, no. 16, pp. 1137-1141, 1998.

[31] J. D. Fowler, S. Virji, R. B. Kaner, and B. H. Weiller, "Hydrogen detection by polyaniline nanofibers on gold and platinum electrodes," Journal of Physical Chemistry C, vol. 113, no. 16, pp. 6444-6449, 2009.

[32] S. Srivastava, S. S. Sharma, S. Kumar, S. Agrawal, M. Singh, and Y. K. Vijay, "Characterization of gas sensing behavior of multi walled carbon nanotube polyaniline composite films," International Journal of Hydrogen Energy, vol. 34, no. 19, pp. 8444-8450, 2009.

[33] M. Matsuguchi, T. Yamanaka, M. Yoshida, S. Kojima, and S. Okumura, "Long-term stability of humidity sensor using polyaniline blend films upon DC operation," Journal of the Electrochemical Society, vol. 156, no. 10, pp. J299-J302, 2009.

[34] M. L. Singla, S. Awasthi, and A. Srivastava, "Humidity sensing; using polyaniline/ $\mathrm{Mn}_{3} \mathrm{O}_{4}$ composite doped with organic/inorganic acids," Sensors and Actuators B, vol. 127, no. 2, pp. 580-585, 2007.

[35] F.-W. Zeng, X.-X. Liu, D. Diamond, and K. T. Lau, "Humidity sensors based on polyaniline nanofibres," Sensors and Actuators B, vol. 143, no. 2, pp. 530-534, 2010. 

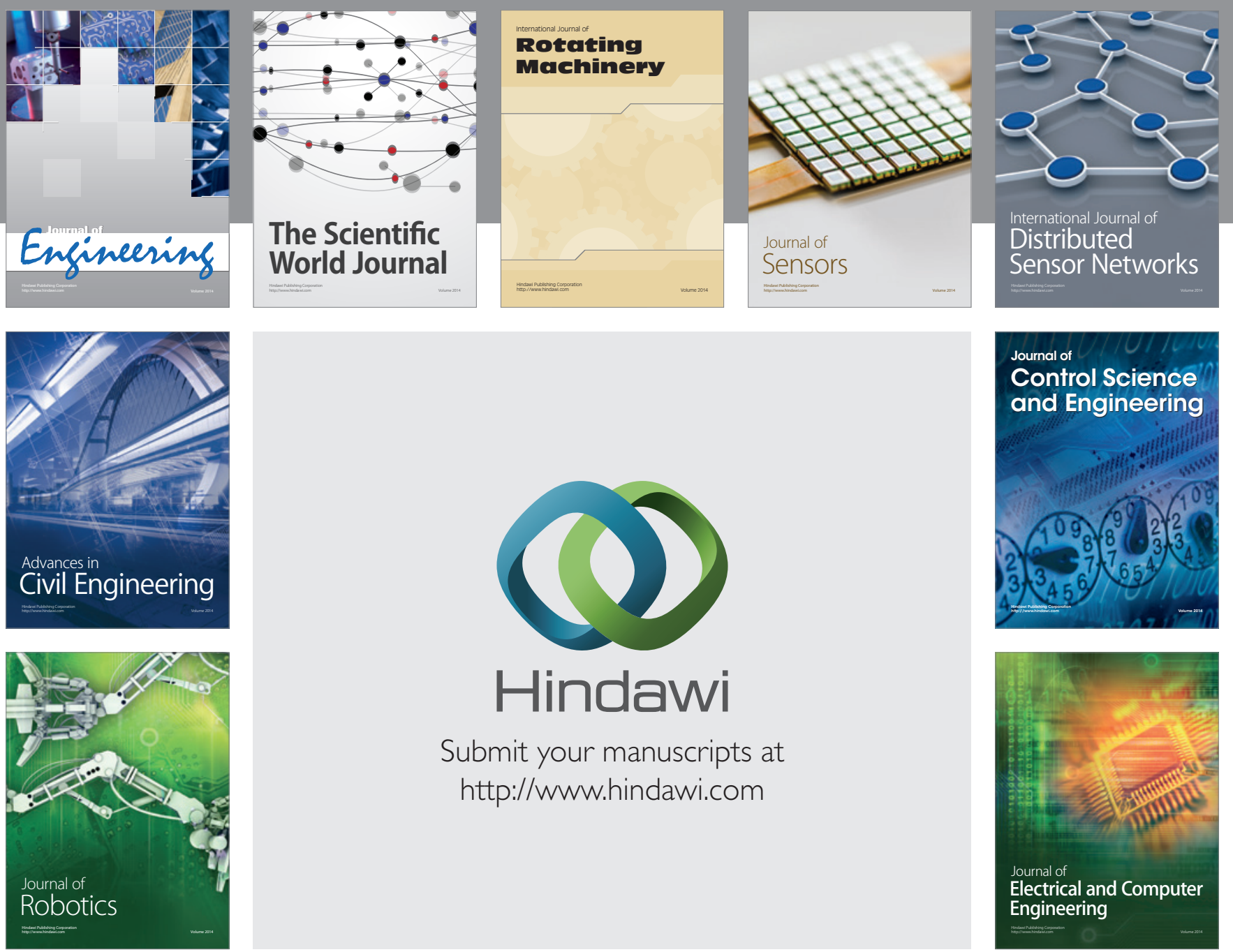

Submit your manuscripts at

http://www.hindawi.com
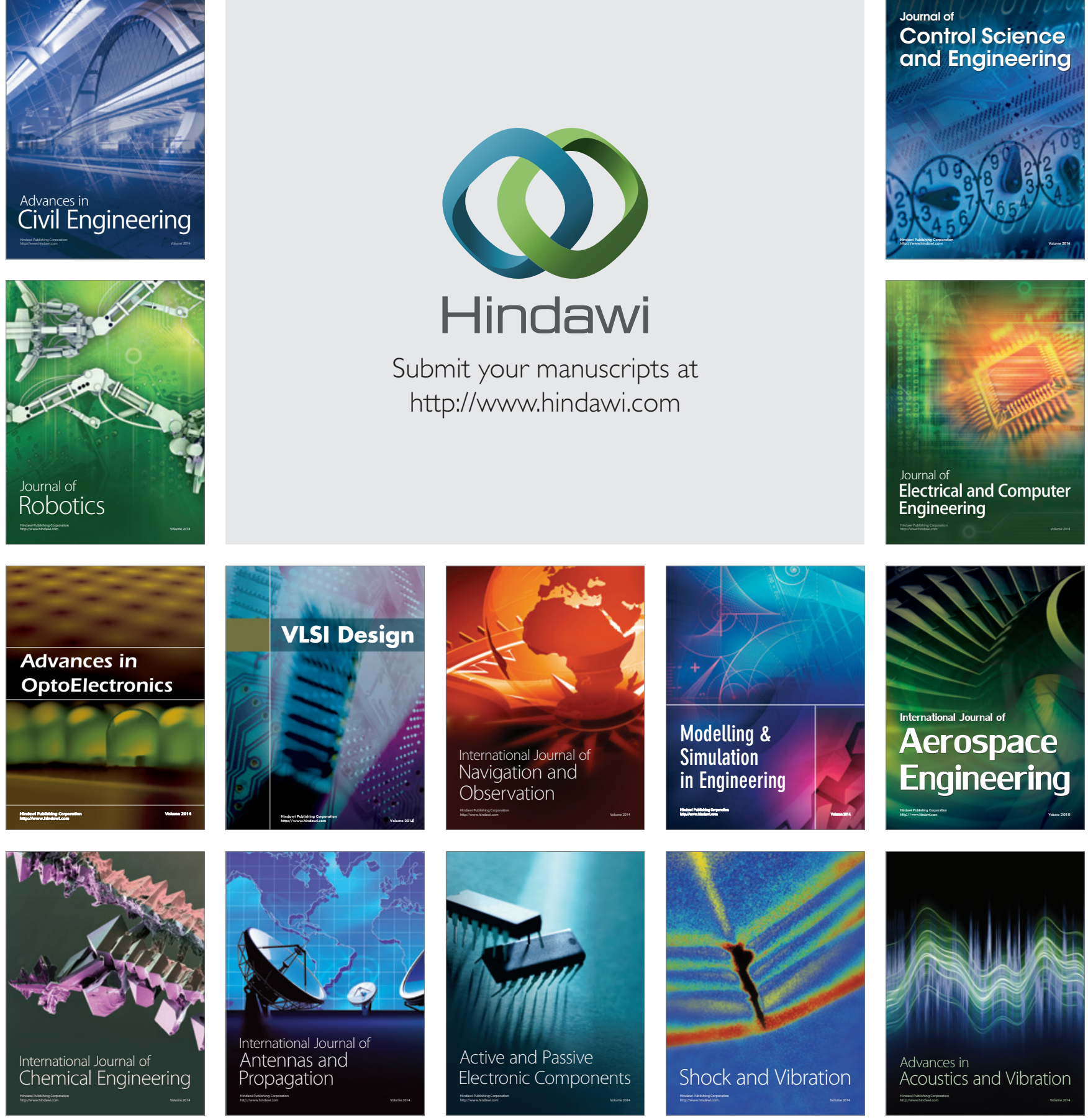